\title{
Partial Encryption on SPIHT Compressed Images
}

\author{
Nidhi Taneja ${ }^{1}$, Balasubramanian Raman ${ }^{2}$, and Indra Gupta ${ }^{1}$ \\ 1 Department of Electrical Engineering, IIT Roorkee, India \\ nidhi.iitr@gmail.com, indrafee@iitr.ernet.in \\ 2 Department of Mathematics, IIT Roorkee, India \\ balarfma@iitr.ernet.in
}

\begin{abstract}
Recently, Multimedia security paradigm has shifted towards encryption techniques combined with compression systems to meet the constraints of wireless networks. Several techniques of encryption with compression have been proposed to reduce processing time, but either they are insecure or computationally intensive. An efficient partial encryption technique based on SPIHT compressed bitstream is thus proposed to provide an efficient encryption for digital images. A satisfactory level of security is achieved with encryption of less than $0.5 \%$ of data for image encoded at $0.8 \mathrm{bpp}$ by encrypting the bits as well as their locations. The proposed technique has large key space and can withstand approximation and brute force attacks.
\end{abstract}

\section{Introduction}

The past decade has witnessed an intensified growth in the development of joint compression and encryption techniques. Traditionally, multimedia data is compressed using a suitable compression algorithm followed by encryption of compressed output with an independent encryption algorithm. Selective encryption is mostly used to provide faster encryption by encrypting only a small portion of multimedia data, either from the final results or from the intermediate steps of compression system. This process must then be reversed by the decoder.

Methods have been proposed to combine partial encryption and compression to reduce the processing time, but either they are insecure or computationally intensive. Thus, a SPIHT based partial encryption technique is proposed that provides adequate security and consumes less computational resources. The proposed algorithm encrypts only the bit values, however, the encrypted bit locations can also be encrypted, providing a two tier security. Analysis has shown that the proposed algorithm gives a good perceptual degradation, low PSNR (Peak Signal to Noise ratio) value, a large key space, and can withstand brute force and approximation attacks. Subsequent section discusses the existing Set partitioning in Hierarchical Tree (SPIHT) based encryption techniques, proposed algorithm and its security analysis. 


\section{Existing SPIHT Based Encryption Techniques}

Most of the compression algorithms generally decompose their input into important and unimportant parts to facilitate efficient encoding. Efficient encryption of only important part makes it difficult for a cryptanalyst to retrieve the correct image from the unencrypted data alone.

Cheng et al. 1] perceived that wrong initial information of two highest pyramid levels will disturb the entire tree structure formed and proposed to encrypt threshold level $n$ and significant information of two highest pyramid levels in the SPIHT [2] encoded bitstream. Said 3] has argued that increased dependency does not necessarily mean high security. Said proved that an acceptable quality of image can be retrieved if the encrypted image and its respective watermarked thumbnail image is available.

Another partial encryption approach based on Color-SPIHT compression technique is proposed by Martin et al. 4. The author proposed to encrypt all the LIP and LIS significant bits encountered during first $K$ sorting passes. The confidentiality vs. processing overhead can be controlled by choosing $K$ during encoding. Although, encryption time can be reduced by selecting only a part of the LIS bitstream.

Norcen and Uhl [5] proposed random permutation of wavelet coefficients in all the subbands before compression by SPIHT or JPEG2000 encoding. However, compression ratio reduces by $27 \%$ even when the permutation keys are not embedded in the encoded bitstream. Encryption of 3D-SPIHT coded video is proposed by Lian et al. [6] using sign bit encryption and two stages of wavelet coefficient confusion. But, coefficient confusion before encoding adversely affects the compression ratio.

To alleviate the problems incurred in above discussed techniques, a partial encryption technique based on SPIHT compression is proposed that gives high computational efficiency without adversely affecting the compression performance.

\section{Proposed Scheme for Image Encryption}

SPIHT generates an encoded bitstream which gives information regarding sign bits, refinement bits, significance of pixels, and significance of sets. The SPIHT encoded bitstream can be divided into three small bitstreams, denoting the bits belonging to LIP, LIS and LSP. The LIP bits denotes the ordered set of bits obtained during the first phase of sorting pass, where coefficients in LIP are tested for significance, LIS denotes the ordered set of bits obtained during the second phase of sorting pass where entire trees are tested for significance, and LSP denotes the ordered set of bits obtained during the refinement pass.

The decoder should consider every bit correctly to faithfully retrieve the image. A slight change in this encoded bitstream disrupt the tree structure, giving a wrongly decoded image. It is observed that encryption of sign bits and refinement bits do not contribute much to the security of images. Whereas, encryption of all the significant information consumes a lot of computational resources or time. 
Thus, encryption of few significant bits representing the vertical descendants of LIS coefficients is proposed. This leads to only minor modifications in the tree structure, but degrades the image completely. The main advantage of the proposed scheme is that the number of bits to be encrypted can be controlled by the user to make an efficient trade off between computational resources and security. Secondly, the location of encrypted bits is not fixed and comes out to be different for different images. To provide sufficient security level, encryption is performed using a block cipher instead of stream cipher.

To identify the encrypted bits, a binary vector $V$ is created during encoding, where $V_{j}=1$ simply depicts that $j^{t h}$ bit in the encoded bitstream represents

SPIHT encoded bitstream

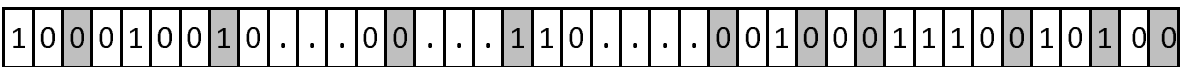

Vector $V$

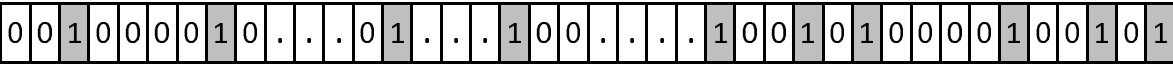

Vertical descendant bits

$$
\begin{array}{|l|l|l|l|l|l|l|l|l|l|l|l|l|l|l|l|l|l|}
\hline 0 & 1 & 0 & 1 & 0 & 0 & 0 & 0 & 1 & 0 & 0 & 1 & 0 & 0 & 0 & 1 & . & . \\
\hline
\end{array}
$$

AES encrypted vertical descendant bits

$$
\begin{array}{|l|l|l|l|l|l|l|l|l|l|l|l|l|l|l|l|l|l|}
\hline 0 & 0 & 0 & 0 & 1 & 1 & 0 & 0 & 1 & 1 & 1 & 1 & 0 & 0 & 0 & 0 & . & . \\
\hline
\end{array}
$$

Fig. 1. Systematic diagram for encryption
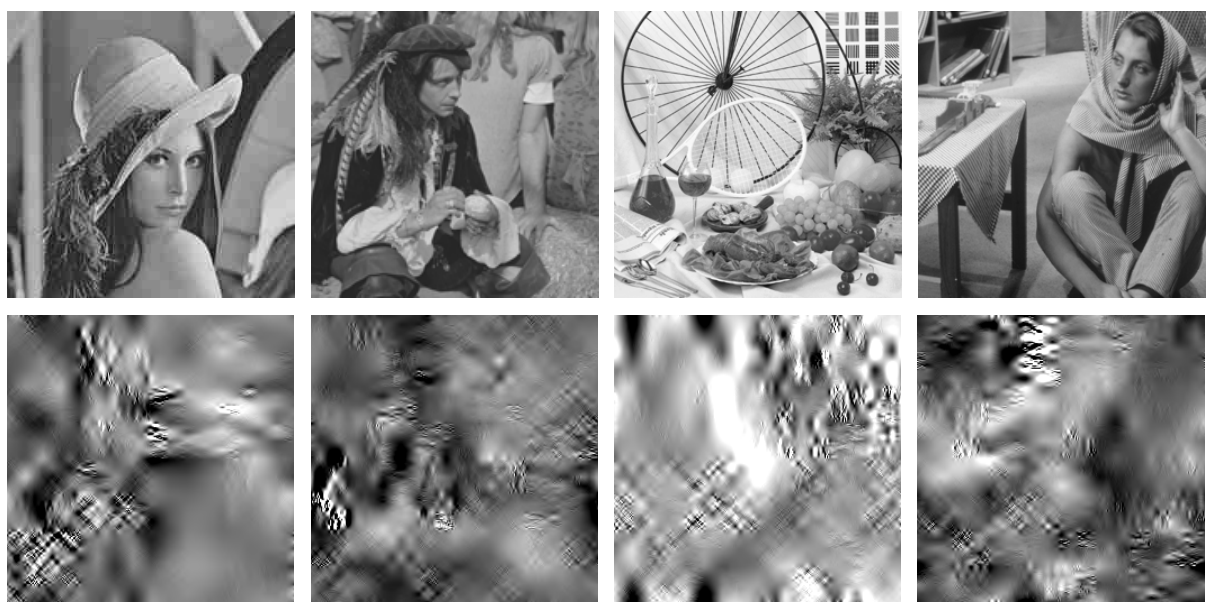

Fig. 2. Results for test images (left to right: lena, pirate, mix, barbara), first row: original image, second row: their encrypted counterparts 
a vertical descendant bit. From the encoded bitstream, $x$ number of bits for which $V_{j}=1$ are grouped together, where $x$ denotes the number of bits to be encrypted and controlled during encoding. These selected bits are then encrypted using AES, and placed back at their original positions in the encoded bitstream. The reconstructed image by decoding of this encrypted bitstream is completely incomprehensible. A systematic diagram depicting the encryption procedure is shown in fig. 1 and the results obtained by encryption are shown in fig. 2 .

Other than the key used during AES, the vector $V$ also becomes a key for the decryption process and transmitted along with the encrypted bitstream. Vector $V$ is a variable length vector, different for different images, and also varies for different decomposition levels or threshold value in case of same image. This vector $V$ can be encoded using (run, EOP) symbols which can further be protected by any public key encryption process. For decryption, the encrypted bits are selected from the encoded bitstream by comparison with the received vector $V$. Reverse process is carried out to reconstruct the original image.

\section{Results and Discussions}

An ideal encryption scheme should (a) ensure no information leakage from the cipher image (b) exhibit high key sensitivity, and (c) be able to withstand various cryptanalytic attacks. To analyze the security of the proposed technique, certain experiments on various grayscale images are perfromed. Fig. 2 shows the cipher images obtained after AES encryption of first 128 vertical descendants bits (less than $0.5 \%$ of the compressed bitstream).

The amount of degradation produced by the employed encryption technique is measured using Peak Signal to Noise Ratio (PSNR). The proposed encryption technique gives a PSNR value between $7 \mathrm{~dB}-15 \mathrm{~dB}$, which is well within the satisfactory limits. The PSNR value obtained for various grayscale images are shown in table 1.

Table 1. PSNR values obtained

cameraman lena pirate barbara woman peppers huts wheel mix montage butterfly

$\begin{array}{lllllllllll}9.89 & 12.29 & 13.09 & 12.52 & 14.19 & 8.75 & 10.80 & 12.44 & 9.96 & 7.84 & 13.76\end{array}$

During analysis, it is observed that in order to get first 128 vertical descendant bits, average length of vector $V$ comes out to be 1500 . In order to break the cipher, an intruder needs to know these encrypted bit locations which require ${ }^{1500} C_{128}$ i.e. 1372 ! attempts. Even after knowing the bit locations, an opponent requires AES key to correctly decrypt the image. Collectively, a large key space is generated in the proposed cryptosystem, which is sufficient enough to resist brute force attacks. 

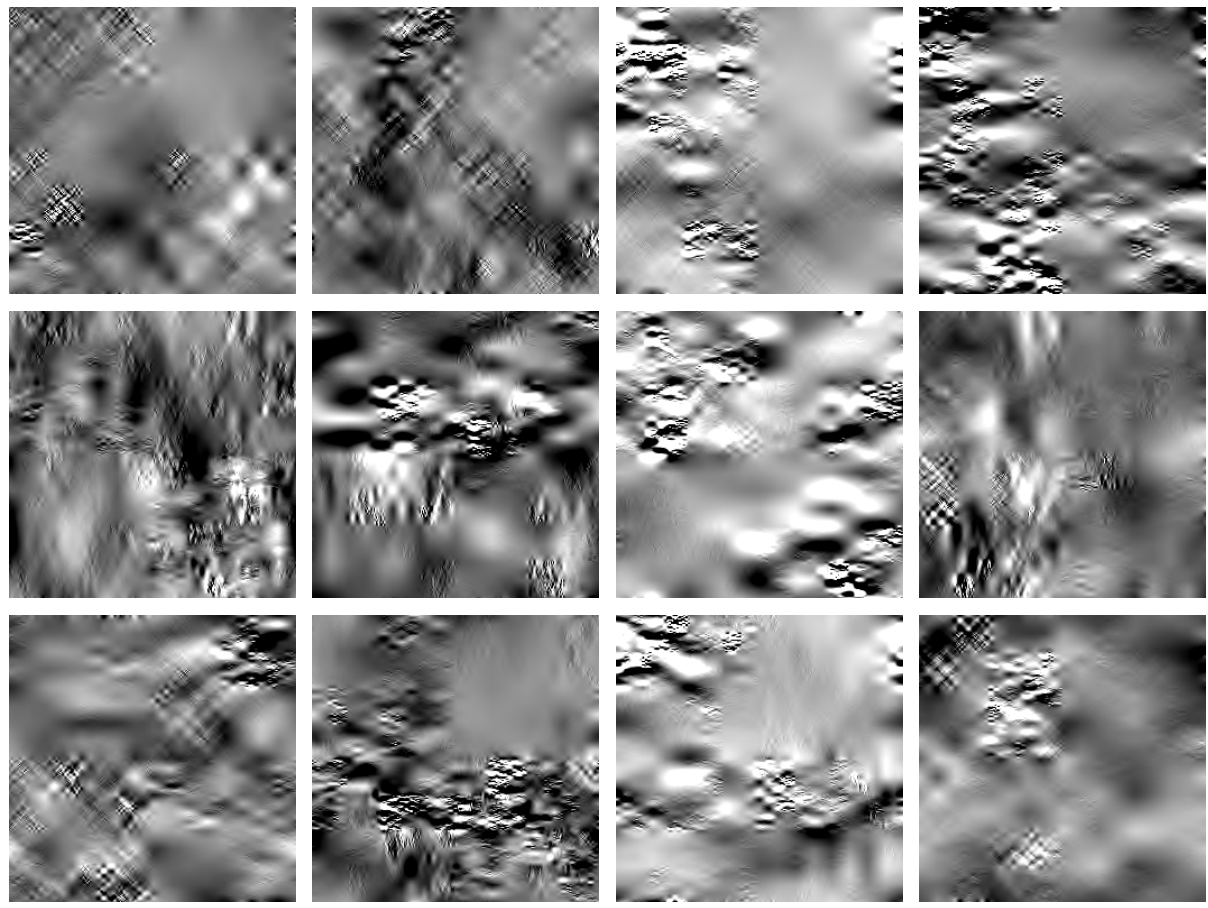

Fig. 3. Results for decrypted images (left to right: lena, pirate, mix, barbara), first row: wrong AES key (only single bit change in encryption and decryption key), second row: wrong vector $V$ (only 5 bit locations are wrongly received), last row: approximation attack on the image (few encrypted values are replaced by 0 or 1 , then decrypted and decoded)

The key sensitive nature of the proposed algorithm is tested by slightly varying the key used during encryption. In the first case, single bit change in the AES key at the LSB position is introduced. And, in the second case, the vector $V$ used during decryption is changed. Randomly five bit locations are made different from the actual vector $V$. Fig. 3 shows the obtained result in both the cases and it can be seen that decryption using wrong key gives totally incomprehensible image.

The strength of encryption technique is also verified by launching approximation attack [7] in which all encrypted bit positions are replaced by a constant value i.e. 0's or 1's. The retrieved degraded images after approximation attack (fig. 3(last row)) do not reveal any information about the original image, thus, verifying the strength of proposed technique.

Use of AES encryption makes the proposed technique scalable in nature with first $n$ bits forming the base layer, protected by a strong cipher. The rest of the bitstream constitute the enhancement layer providing bit level scalability. This enhancement layer can also be encrypted using a lightweight encryption scheme like XOR function. 


\subsection{Comparison with Existing Techniques}

The proposed scheme is different from the scheme in 14 in three important ways: (a) computational overhead is reduced by encrypting only vertical descendant depicting bits of LIS coefficients, (b) the level of confidentiality achieved vs. computational overhead can be controlled by controlling the number of bits to be encrypted, and (c) encryption of vector $V$ provides an additional security level. Further, the schemes in 516 are implemented before encoding, which adversely affects the compression ratio whereas the proposed technique is implemented on compressed bitstream without affecting the compression efficiency.

\section{Conclusion}

This paper proposes a partial encryption approach that encrypts selected data from the SPIHT compressed bitstream. Security is achieved by not only encrypting selected part of compressed bitstream, but can also be increased by encrypting the bit locations of encrypted bits. A good trade-off between security and computational resources is achieved without adversely affecting the compression ratio. The proposed technique provides a large key space, sufficient level of perceptual degradation, can withstand approximation attacks and brute force attacks.

\section{References}

1. Cheng, H., Li, X.: Partial encryption of compressed images and videos. IEEE Trans. Signal Process. 48(8), 2439-2451 (2000)

2. Said, A., Pearlman, W.A.: A new, fast, and efficient image codec based on set partitioning in hierarchical trees. IEEE Trans. Circuits \& Systems Video Tech. 6, 243-250 (1996)

3. Said, A.: Measuring the strength of partial encryption schemes. In: IEEE Int. Conf. Image Process., vol. 2, pp. 1126-1129 (2005)

4. Martin, K., Lukac, R., Plataniotis, K.N.: Efficient encryption of wavelet-based coded color images. Patt. Recogn. 38, 1111-1115 (2005)

5. Norcen, R., Uhl, A.: Encryption of wavelet-coded imagery using random permutations. In: Proc. IEEE Int. Conf. Image Process., pp. 3431-3434 (2004)

6. Lian, S., Sun, J., Wang, Z.: A secure 3D-SPIHT codec. In: Proc. European Signal Process. Conf., pp. 813-816 (2004)

7. Mao, Y., Wu, M.: A joint signal processing and cryptographic approach to multimedia encryption. IEEE Trans. Image Process. 15(7), 2061-2075 (2006) 